\title{
Induction of final maturation by sperm penetration in canine oocytes
}

\author{
M. Saint-Dizier ${ }^{1}$, J-P. Renard ${ }^{3}$ and S. Chastant-Maillard ${ }^{1,2 *}$ \\ ${ }^{1}$ Unit of Reproductive Biology, Veterinary School of Alfort, 94704 Maisons-Alfort, France; \\ ${ }^{2}$ Service of Reproduction, Veterinary School of Alfort, 94704 Maisons-Alfort, France; and \\ ${ }^{3}$ Unité de Biologie du Développement, Institut National de la Recherche Agronomique \\ (INRA), 78352 Jouy-en-Josas, France
}

In contrast to oocytes of most mammals, the canine oocyte is at the germinal vesicle stage at ovulation. Moreover, the bitch is receptive to mating while immature oocytes are present in the oviducts. The aims of this study were to examine the influence of fertilization in immature oocytes on the resumption of meiosis, and the modification of both male and female chromatin in fertilized oocytes. Canine cumulus-oocyte complexes collected from routine ovariectomies were cultured in medium 199 with $20 \%$ fetal calf serum for $24 \mathrm{~h}$, incubated in the same medium with fresh semen for $24 \mathrm{~h}$, washed, cultured for a further $24 \mathrm{~h}$ and fixed. Control oocytes were cultured in the same medium but without spermatozoa for 24, 48 or $72 \mathrm{~h}$. After fixation, chromatin was stained with propidium iodide and examined using laser scanning confocal microscopy. The data indicate that sperm penetration can occur in immature canine oocytes and that it induces resumption of meiosis. After $72 \mathrm{~h}$ of culture, the percentage of oocytes at the germinal vesicle stage was significantly lower in fertilized oocytes $(40 \%$ versus $\mathbf{6 0 . 3 \%}$ for control oocytes; $P<\mathbf{0 . 0 5}$ ) and the percentage of oocytes beyond metaphase I was significantly greater in fertilized oocytes $(28.3 \%$ metaphase I and II, and two pronuclei versus $\mathbf{1 0 . 2} \%$ metaphase I and II for control oocytes; $P<0.01)$. Observation and measurement of the area of chromatin in fertilized oocytes showed an overall parallel condensation-decondensation of both female and male chromatin from the germinal vesicle stage to the pronuclear stage.

\section{Introduction}

Recent studies have attempted to improve the in vitro maturation of canine oocytes using various culture conditions (Cinone et al., 1992; Robertson et al., 1992; Hewitt and England, 1997a, 1999; Hewitt et al., 1998). However, the efficiency of in vitro maturation of canine oocytes remains very low compared with that of other domestic species. After $72-96 \mathrm{~h}$ of culture, the percentage of oocytes at metaphase II ranges from 0\% (Robertson et al., 1992; Hewitt and England, 1999) to 32-42\% (Nickson et al., 1993; Yamada et al., 1993), regardless of the endocrine status of the bitch at oocyte recovery (Hewitt and England, 1997a). Improvement of in vitro maturation and fertilization remains essential for the development of assisted reproduction technologies in canine reproduction.

The low efficiency of in vitro maturation of oocytes may be related to the physiology of bitches. In most mammals, oocyte maturation occurs within the ovarian follicle and the first meiotic division takes place at ovulation. Oocytes arrested at metaphase II are ready to be fertilized within

*Correspondence

Email: chastant@vet-alfort.fr the oviduct. In contrast, canine (bitch, vixen) oocytes are ovulated as immature oocytes at the germinal vesicle stage and expel the first polar body at 48-72 h after ovulation when oocytes are in the middle part of the oviduct (Van der Stricht, 1923; Pearson and Enders, 1943; Tsutsui and Shimazu, 1975; Tsutsui, 1989).

The extended period in the oviduct required for oocyte maturation raises the question of the maturation stage at the time of fertilization in vivo. Bitches are receptive to mating at the time of the LH peak, which occurs 36-50 h before ovulation (Concannon et al., 1989). During a natural mating, spermatozoa can reach the oviducts 25-120 s after ejaculation (Evans, 1933) and motile spermatozoa can be found in the lumen of the uterus up to 4-6 days after copulation (Doak et al., 1967). Since complete nuclear maturation of canine oocytes takes place during 2-3 days within the oviducts, spermatozoa are already present around cumulus-oocyte complexes before the initiation of maturation. This situation differs from that in most other mammals, in which spermatozoa are present around cumulus-oocyte complexes in vivo after the oocytes have reached metaphase II. It remains to be established whether canine oocytes can be fertilized at immature stages in vivo. Although it is generally considered that canine oocytes 
cannot be fertilized before metaphase II (Gier, 1950; Holst and Phemister, 1971; Tsutsui, 1989), one in vivo study reported the presence of sperm heads in oocytes at germinal vesicle and metaphase I stages (Van der Stricht, 1923). Results of in vitro fertilization experiments also indicate that the maturation stage at the time of fertilization does not modify the sperm penetration rate (Mahi and Yanagimachi, 1976; Yamada et al., 1993; Hewitt and England, 1997b). Effects of sperm penetration on the onset or the completion of canine oocyte maturation remain to be determined.

In such cases of premature fertilization in bitches, data concerning the relationship between the maturation stage of the oocyte and the sperm head aspect within the cytoplasm are limited and contradictory. Van der Stricht (1923) reported very little modification of the penetrated sperm head until the expulsion of the second polar body. Mahi and Yanagimachi (1976) observed swelling of the sperm nuclei in the cytoplasm of oocytes at all maturation stages with no difference in the degree of decondensation. Yamada et al. (1992) observed an enlarged sperm head in oocytes arrested at the germinal vesicle stage, but a male pronucleus was observed only in oocytes that had a female pronucleus. Renton et al. (1991) observed two structures in a one-cell embryo recovered 5 days after assumed ovulation; these two structures were determined to be pronuclei, but no further details were supplied. Finally, an electron microscopy study of fox embryos fertilized in vivo did not report a relationship between the maternal meiotic stage and the stage of paternal pronucleus formation (Farstad et al., 1993).

The aims of this study were to (i) investigate whether sperm penetration in immature oocytes initiates the maturation process in vitro, and (ii) examine the modification to the sperm nucleus after penetration in relation to the meiotic stage of the oocyte.

\section{Materials and Methods}

\section{Oocyte collection and culture}

Ovaries were obtained from pubertal bitches during routine ovariectomies at the veterinary hospital of Alfort. Ovaries were placed immediately in PBS at $38^{\circ} \mathrm{C}$. Within $2 \mathrm{~h}$ after surgery, each ovary was dissected from fat and vessels, rinsed in PBS and placed in a culture dish containing $1 \mathrm{ml}$ TCM 199 (Gibco BRL, Cergy-Pontoise) with Hepes and $20 \%$ fetal calf serum (FCS, Dutcher, Brumath) for further dissection. Ovarian tissue was sliced repeatedly to release cumulus-oocyte complexes. Cumulus-oocyte complexes that had more than three layers of cumulus cells and dark pigmented oocyte cytoplasm were cultured in groups of $10-30$ in $500 \mu$ d droplets of TCM 199 supplemented with $20 \%$ fetal calf serum at $38.5^{\circ} \mathrm{C}$ in a humidified atmosphere of $5 \% \mathrm{CO}_{2}$ in air for 24-72 $\mathrm{h}$.

\section{Sperm preparation and IVF}

One healthy beagle dog was used as a semen donor.
The first and second fractions of the ejaculate were collected separately by manual stimulation. Only the spermrich fraction (that is, the second fraction) was used for IVF and was stored with no treatment at room temperature before use. Semen was assessed for sperm motility using optical microscopy. Samples with $>80 \%$ motile spermatozoa were used for IVF. Sperm concentration was evaluated using a Thoma chamber.

After 24 h of culture, groups of 20-30 cumulus-oocyte complexes were incubated with spermatozoa at a final concentration of $10^{6}$ motile spermatozoa $\mathrm{ml}^{-1}$ in fresh culture medium. After $24 \mathrm{~h}$ of incubation with spermatozoa, cumulus-oocyte complexes were rinsed in TCM 199 supplemented with $20 \%$ fetal calf serum and cultured in fresh medium for a further $24 \mathrm{~h}$. A total culture period of $72 \mathrm{~h}$ was chosen arbitrarily for the determination of maturation stages of fertilized oocytes. Control batches of cumulus-oocyte complexes were cultured without spermatozoa in the same culture medium for 24,48 or $72 \mathrm{~h}$. For control oocytes cultured for 48 or 72 h, all cumulusoocyte complexes were placed in fresh medium at $24 \mathrm{~h}$ intervals.

\section{Chromatin staining}

At the end of the culture period, control (24, 48 and $72 \mathrm{~h})$ and fertilized (72 h) oocytes were denuded mechanically using a finely drawn glass pipette and fixed in paraformaldehyde $\left(2.5 \% \mathrm{w} / \mathrm{v}\right.$ in PBS) for $30 \mathrm{~min}$ at $37^{\circ} \mathrm{C}$. Fixed oocytes were stored in PBS for $0-3$ days at $4^{\circ} \mathrm{C}$ until used for chromatin staining.

DNA was stained by exposure to $10 \mu \mathrm{g}$ propidium iodide $\mathrm{ml}^{-1}$ (Sigma, St Quentin Fallavier) in PBS supplemented with $2 \%$ fetal calf serum and $0.5 \%$ Triton-X100 $(w / v)$. Chromatin status was determined using laser scanning confocal microscopy (CLSM 310, Karl Zeiss). In a preliminary study, the reliability of Hoechst staining and fluorescence microscopy was compared with propidium iodide (w/v) staining and laser scanning confocal microscopy after 24, 48 and $72 \mathrm{~h}$ of culture. Propidium iodide staining and confocal microscopy were used in this study as the detection rate of both germinal vesicles and germinal vesicle breakdown stages was significantly increased compared with the alternative method.

The area of male and female chromatin was measured at the largest diameter of the nucleus using the Imagetool software (UTHSCSA, San Antonio). Images used for the area measurements were saved from serial sections of each embryo performed using scanning confocal microscopy.

\section{Microtubule staining}

The organization of cytoplasmic microtubules is closely associated with oocyte nuclear maturation in dogs (M. SaintDizier, J-P. Renard and S. Chastant-Maillard, unpublished) and other species (Kim et al., 1996, 1998; Dedieu et al., 1997). For that reason, staining of both microtubules and chromatin was used for the determination of maturation 
stages. Microtubules were visualized by immunocytochemistry according to a technique used in rabbits (Adenot et al., 1997). After oocytes were fixed and washed three times in PBS, they were incubated in a blocking solution (PBS containing $20 \%$ fetal calf serum and $0.5 \%$ Triton-X100) for 30 min at $37^{\circ} \mathrm{C}$ and subsequently incubated for $60 \mathrm{~min}$ at $37^{\circ} \mathrm{C}$ with mouse $\alpha$-tubulin monoclonal antibody (Sigma) diluted $1: 200$ in PBS containing $2 \%$ fetal calf serum and $0.5 \%$ Triton-X100. After three washes in PBS containing 2\% fetal calf serum and $0.5 \%$ Triton-X100, oocytes were incubated for $60 \mathrm{~min}$ at $37^{\circ} \mathrm{C}$ in fluorescein isothiocyanate (FITC)-labelled goat anti-mouse antibody (Sigma) diluted 1:400 in PBS containing $2 \%$ fetal calf serum and $0.5 \%$ Triton- $\mathrm{X} 100$. After oocytes were washed three times in PBS containing 2\% fetal calf serum and $0.5 \%$ Triton-X100, chromatin was stained as described above. Stained oocytes were mounted under a coverslip with Vectashield (Vector Lab, Burlingame). The slides were examined under a laser scanning confocal microscope.

\section{Statistical analysis}

Percentages of non-degenerated oocytes at different maturation stages were compared by chi-squared analysis and Student's $t$ test. Differences between values were considered statistically significant when $P<0.05$. Chromatin areas were analysed by ANOVA followed by Student-Newman-Keuls' test (SAS software, SAS Institute NY, 1996).

\section{Results}

\section{In vitro maturation}

The determination of maturation stages at 24, 48 and $72 \mathrm{~h}$ was possible for $98.0 \%$ of non-degenerated control oocytes $(n=665) \quad$ (Fig. 1). Oocyte degeneration was observed as pycnosis, no identifiable intracytoplasmic structures, or loss of the cytoplasmic membrane. The de- generation rate did not change significantly between 24, 48 and $72 \mathrm{~h}$ and was on average $28.9 \%(n=936)$ (Table 1). Parthenogenetic activation was not observed.

Between 24 and $48 \mathrm{~h}$ of culture, the percentage of oocytes at the germinal vesicle stage decreased $(P<0.05)$, whereas the percentage of oocytes at the germinal vesicle breakdown stage increased $(P<0.05)$. During the same culture period, the proportion of oocytes at metaphase I and II remained constant $(8.4 \%$ and $9.4 \%$ at metaphase I and II at 24 and $48 \mathrm{~h}$, respectively; $P>0.05$ ) (Table 1 ). Between 48 and $72 \mathrm{~h}$ of culture, the percentage of oocytes at different maturation stages remained unchanged. Initiation of in vitro maturation was observed for $36.6 \%$ ( $n=380)$ of oocytes during the first $48 \mathrm{~h}$ of culture.

\section{Effect of sperm penetration on oocyte maturation in vitro}

Maturation rates of fertilized oocytes compared with control oocytes at $72 \mathrm{~h}$ of culture are shown (Table 2). Sperm penetration resulted in a significant decrease in the percentage of oocytes at the germinal vesicle stage $(P<0.05)$ and a significant increase in the percentage of oocytes beyond metaphase I (metaphase II and two pronuclei stages) at $72 \mathrm{~h}$ of culture $(P<0.01)$. After $72 \mathrm{~h}$ of culture, meiosis had been initiated in $53.3 \%$ of penetrated oocytes $(n=60)$ (that is, the oocytes were at the germinal vesicle breakdown stage or beyond) compared with $37.6 \%$ of control oocytes $(n=285)$. However, $40 \%$ of fertilized oocytes remained at the germinal vesicle stage. The proportion of oocytes at the germinal vesicle breakdown stage did not change significantly after sperm penetration. Metaphase I was not observed in fertilized oocytes at $72 \mathrm{~h}$ (Table 2).

Polyspermy was observed in $46.7 \%$ of fertilized oocytes. The average number of penetrated spermatozoa per oocyte for each stage of meiotic maturation is shown (Table 3). Comparison of monospermic oocytes with polyspermic oocytes indicated that polyspermia had no

Table 1. Meiotic maturation of canine oocytes in vitro

\begin{tabular}{|c|c|c|c|c|c|c|c|}
\hline \multirow[b]{2}{*}{$\begin{array}{l}\text { Culture } \\
\text { period } \\
\text { (h) }\end{array}$} & \multirow[b]{2}{*}{$\begin{array}{l}\text { Number of } \\
\text { oocytes } \\
\text { examined }\end{array}$} & \multirow{2}{*}{$\begin{array}{l}\text { Number of } \\
\text { degenerated } \\
\text { oocytes } \\
(\%)\end{array}$} & \multicolumn{5}{|c|}{ Number of oocytes that reached each stage (\%)* } \\
\hline & & & $\begin{array}{l}\text { Germinal } \\
\text { vesicle }\end{array}$ & $\begin{array}{l}\text { Germinal } \\
\text { vesicle } \\
\text { breakdown }\end{array}$ & Metaphase & $\begin{array}{l}\text { Metaphase } \\
\text { II }\end{array}$ & $\begin{array}{c}\text { Unidentifiable } \\
\text { nuclear } \\
\text { material }\end{array}$ \\
\hline 24 & 169 & $\begin{array}{c}50 \\
(29.6)\end{array}$ & $\begin{array}{c}82 \\
(68.9)^{\mathrm{a}}\end{array}$ & $\begin{array}{c}23 \\
(19.3)^{\mathrm{a}}\end{array}$ & $\begin{array}{c}8 \\
{[(6.7)}\end{array}$ & $\begin{array}{c}2 \\
(1.7)]^{\mathrm{a}}\end{array}$ & $\begin{array}{c}4 \\
(3.4)\end{array}$ \\
\hline 48 & 391 & $\begin{array}{c}130 \\
(33.2)\end{array}$ & $\begin{array}{c}154 \\
(59.0)^{b}\end{array}$ & $\begin{array}{c}81 \\
(31.0)^{b}\end{array}$ & $\begin{array}{c}19 \\
{[(7.3)}\end{array}$ & $\begin{array}{c}6 \\
(2.1)]^{\mathrm{a}}\end{array}$ & $\begin{array}{c}3 \\
(1.1)\end{array}$ \\
\hline 72 & 376 & $\begin{array}{c}91 \\
(24.2)\end{array}$ & $\begin{array}{c}172 \\
(60.3)^{b}\end{array}$ & $\begin{array}{c}78 \\
(27.4)^{b}\end{array}$ & $\begin{array}{c}23 \\
{[(8.1)}\end{array}$ & $\begin{array}{c}6 \\
(2.1)]^{a}\end{array}$ & $\begin{array}{c}6 \\
(2.1)\end{array}$ \\
\hline
\end{tabular}

*Percentage of non-degenerated oocytes.

${ }^{a b}$ For each stage, values with different superscripts are significantly different $(P<0.05)$.

Values in square brackets were combined to achieve sufficient numbers for statistical analysis. 

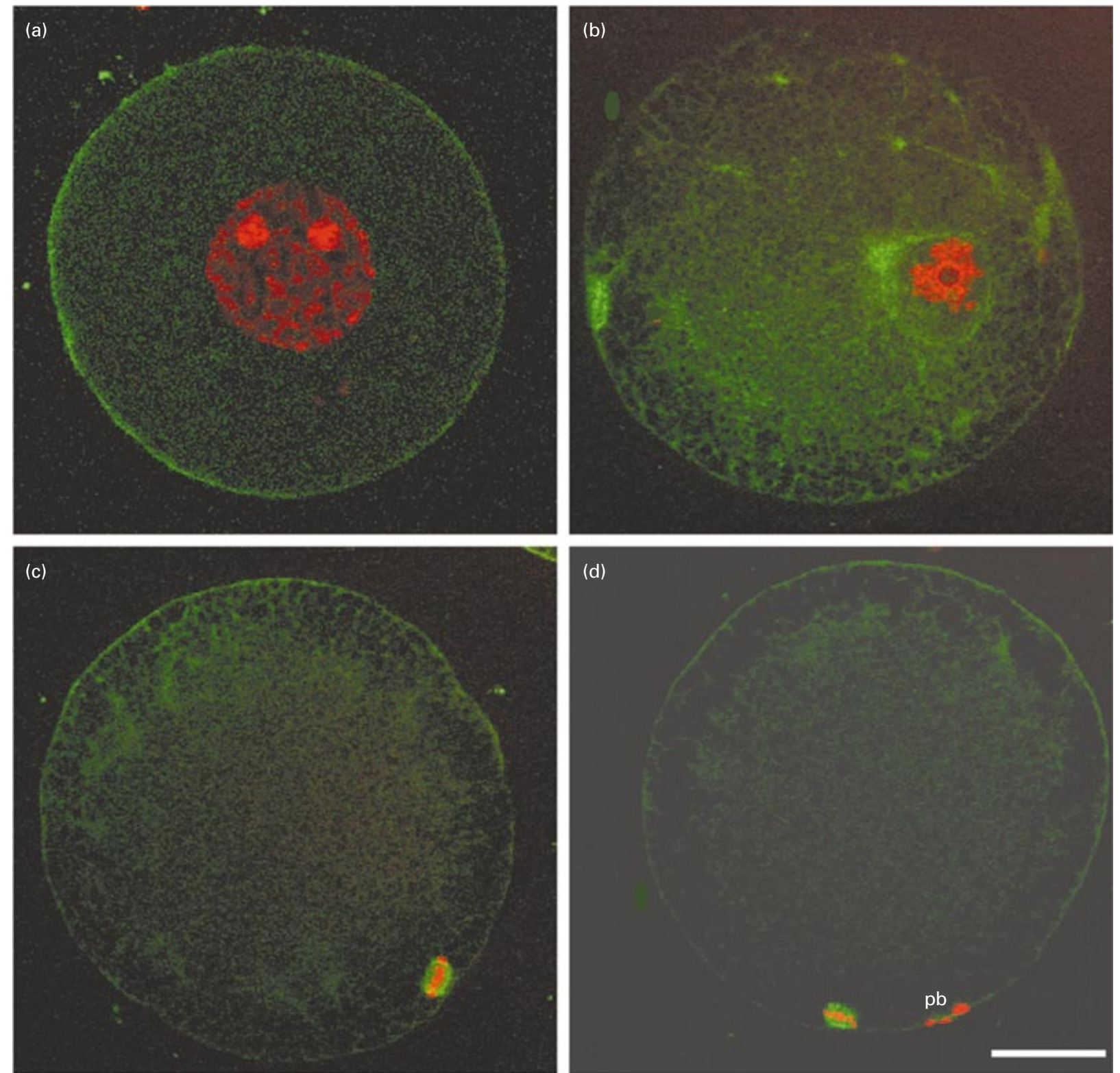

Fig. 1. Canine oocytes after $72 \mathrm{~h}$ of in vitro culture, examined using laser scanning confocal microscopy (control oocytes). (a) Germinal vesicle; (b) germinal vesicle breakdown; (c) metaphase I; and (d) metaphase II. pb: polar body. Scale bar represents $30 \mu \mathrm{m}$.

effect on the rate of maturation. In polyspermic oocytes, the number of penetrating spermatozoa (ranging from one to 12 per oocyte) was not correlated with the maturation stage (Table 3 ). Thus the number of penetrating spermatozoa did not affect maturation of fertilized oocytes.

\section{Evolution of male and female chromatin areas}

The status of the male chromatin during the completion of the female meiotic process was determined. In fertilized oocytes at the germinal vesicle stage, the intracytoplasmic male chromatin expanded in contrast to the sperm heads attached to the zona pellucida (Fig. 2a). From the germinal vesicle stage to the germinal vesicle breakdown stage, the female chromatin underwent significant condensation, whereas no change was observed in the male chromatin (Fig. 2b). At metaphase II, male and female chromatin were observed as several groups of chromosomes, each located on microtubule spindles (Fig. 2c). At this stage, female chromatin could not be distinguished from male chromatin. At the pronuclear stage, both male and female chromatin were decondensed and showed homogeneous DNA staining, except in one to three round areas, which probably corresponded to the nucleoli (Fig. 2d). 
Table 2. Effect of fertilization on the maturation of canine oocytes

\begin{tabular}{|c|c|c|c|c|c|c|c|}
\hline & \multirow{2}{*}{$\begin{array}{l}\text { Number of } \\
\text { oocytes } \\
\text { examined } \\
\text { at } 72 \mathrm{~h}\end{array}$} & \multirow[b]{2}{*}{$\begin{array}{l}\text { Germinal } \\
\text { vesicle }\end{array}$} & \multicolumn{5}{|c|}{ Number of oocytes that reached each stage (\%)* } \\
\hline & & & $\begin{array}{l}\text { Germinal } \\
\text { vesicle } \\
\text { breakdown }\end{array}$ & $\begin{array}{c}\text { Metaphase } \\
\text { । }\end{array}$ & $\begin{array}{l}\text { Metaphase } \\
\text { II }\end{array}$ & $\begin{array}{l}\text { Pronuclear } \\
\text { stage }\end{array}$ & $\begin{array}{c}\text { Unidentifiable } \\
\text { nuclear } \\
\text { material }\end{array}$ \\
\hline $\begin{array}{l}\text { Control } \\
\text { oocytes }\end{array}$ & 285 & $\begin{array}{c}172 \\
(60.3)^{\mathrm{a}}\end{array}$ & $\begin{array}{c}78 \\
(27.4)^{\mathrm{a}}\end{array}$ & $\begin{array}{c}23 \\
{[(8.1)}\end{array}$ & $\begin{array}{c}6 \\
(2.1)\end{array}$ & $\begin{array}{c}- \\
(-)]^{\mathrm{a}}\end{array}$ & $\begin{array}{c}6 \\
(2.1)\end{array}$ \\
\hline $\begin{array}{l}\text { Fertilized } \\
\text { oocytes }\end{array}$ & 60 & $\begin{array}{c}24 \\
(40.0)^{b}\end{array}$ & $\begin{array}{c}15 \\
(25.0)^{\mathrm{a}}\end{array}$ & $\begin{array}{l}- \\
{[(-)}\end{array}$ & $\begin{array}{c}6 \\
(10.0)\end{array}$ & $\begin{array}{c}11 \\
(18.3)]^{b}\end{array}$ & $\begin{array}{c}4 \\
(6.7)\end{array}$ \\
\hline
\end{tabular}

*Percentage of non-degenerated oocytes.

For each maturation stage, values with different superscripts are significantly different $(P<0.05$ : germinal vesicle stage; $P<0.01$ : metaphase I, metaphase II and pronuclear stages).

Values in square brackets were combined to achieve sufficient numbers for statistical analysis.

Table 3. Influence of the number of penetrated spermatozoa on the maturation of canine oocytes

\begin{tabular}{|c|c|c|c|c|c|c|c|}
\hline & $\begin{array}{l}\text { Number of } \\
\text { oocytes } \\
\text { examined } \\
\text { at } 72 \mathrm{~h}\end{array}$ & $\begin{array}{l}\text { Germinal } \\
\text { vesicle }\end{array}$ & $\begin{array}{l}\text { Germinal } \\
\text { vesicle } \\
\text { breakdown }\end{array}$ & $\begin{array}{c}\text { Metaphase } \\
\text { I }\end{array}$ & $\begin{array}{l}\text { Metaphase } \\
\text { II }\end{array}$ & $\begin{array}{l}\text { Pronuclear } \\
\text { stage }\end{array}$ & $\begin{array}{c}\text { Unidentifiable } \\
\text { nuclear } \\
\text { material }\end{array}$ \\
\hline \multicolumn{8}{|c|}{$\begin{array}{l}\text { Number of oocytes } \\
\text { that reached each } \\
\text { stage }(\%)^{*}\end{array}$} \\
\hline $\begin{array}{l}\text { Monospermic } \\
\text { oocytes }\end{array}$ & 32 & $\begin{array}{c}14 \\
(43.7)^{\mathrm{a}}\end{array}$ & $\begin{array}{c}8 \\
(25.0)^{\mathrm{a}}\end{array}$ & $\begin{array}{l}- \\
{[(-)}\end{array}$ & $\begin{array}{c}2 \\
(6.3)\end{array}$ & $\begin{array}{c}5 \\
(15.2)]^{\mathrm{a}}\end{array}$ & $\begin{array}{c}3 \\
(9.4)\end{array}$ \\
\hline $\begin{array}{l}\text { Polyspermic } \\
\text { oocytes }\end{array}$ & 28 & $\begin{array}{c}10 \\
(35.7)^{\mathrm{a}}\end{array}$ & $\begin{array}{c}7 \\
(25.0)^{\mathrm{a}}\end{array}$ & $\begin{array}{l}- \\
{[(-)}\end{array}$ & $\begin{array}{c}4 \\
(14.3)\end{array}$ & $\begin{array}{c}6 \\
(21.4)]^{\mathrm{a}}\end{array}$ & $\begin{array}{c}1 \\
(2.1)\end{array}$ \\
\hline $\begin{array}{l}\text { Number of } \\
\text { spermatozoa } \\
\text { per oocyte } \\
\text { (range) for } \\
\text { polyspermic } \\
\text { oocytes }\end{array}$ & & $\begin{array}{l}3.5 \pm 3.2 \\
(2-12)\end{array}$ & $\begin{array}{c}3.6 \pm 2.4 \\
(2-8)\end{array}$ & - & $\begin{array}{c}3.0 \pm 0.8 \\
(2-4)\end{array}$ & $\begin{array}{c}2.8 \pm 1.2 \\
(2-5)\end{array}$ & 2 \\
\hline
\end{tabular}

For polyspermic oocytes, the number of spermatozoa is indicated as mean $\pm \mathrm{SD}$.

*Percentage of non-degenerated oocytes.

For each maturation stage, values with different superscripts are significantly different $(P<0.05)$.

Values in square brackets were combined to achieve sufficient numbers for statistical analysis.

Measurement of the areas of the male and female chromatin revealed significant modifications according to the stage of oocyte maturation $(P<0.01)$ (Fig. 3). After sperm penetration, a classical evolution of female chromatin was observed: condensation between the germinal vesicle and the germinal vesicle breakdown stages, which was marked at the metaphase stage, and was followed by decondensation at the pronuclear stage. At metaphase, the area of the chromatin was considered to be zero (Fig. 3). At the pronuclear stage, by analogy with other mammals, the smaller pronucleus was considered as female in origin (Sutovsky and Schatten, 1998). The evolution of the male chromatin was generally similar, although condensation between the germinal vesicle and the germinal vesicle breakdown stages was not observed (Fig 3).

\section{Discussion}

The results of this study indicate that sperm penetration can occur in immature canine oocytes and that it induces 

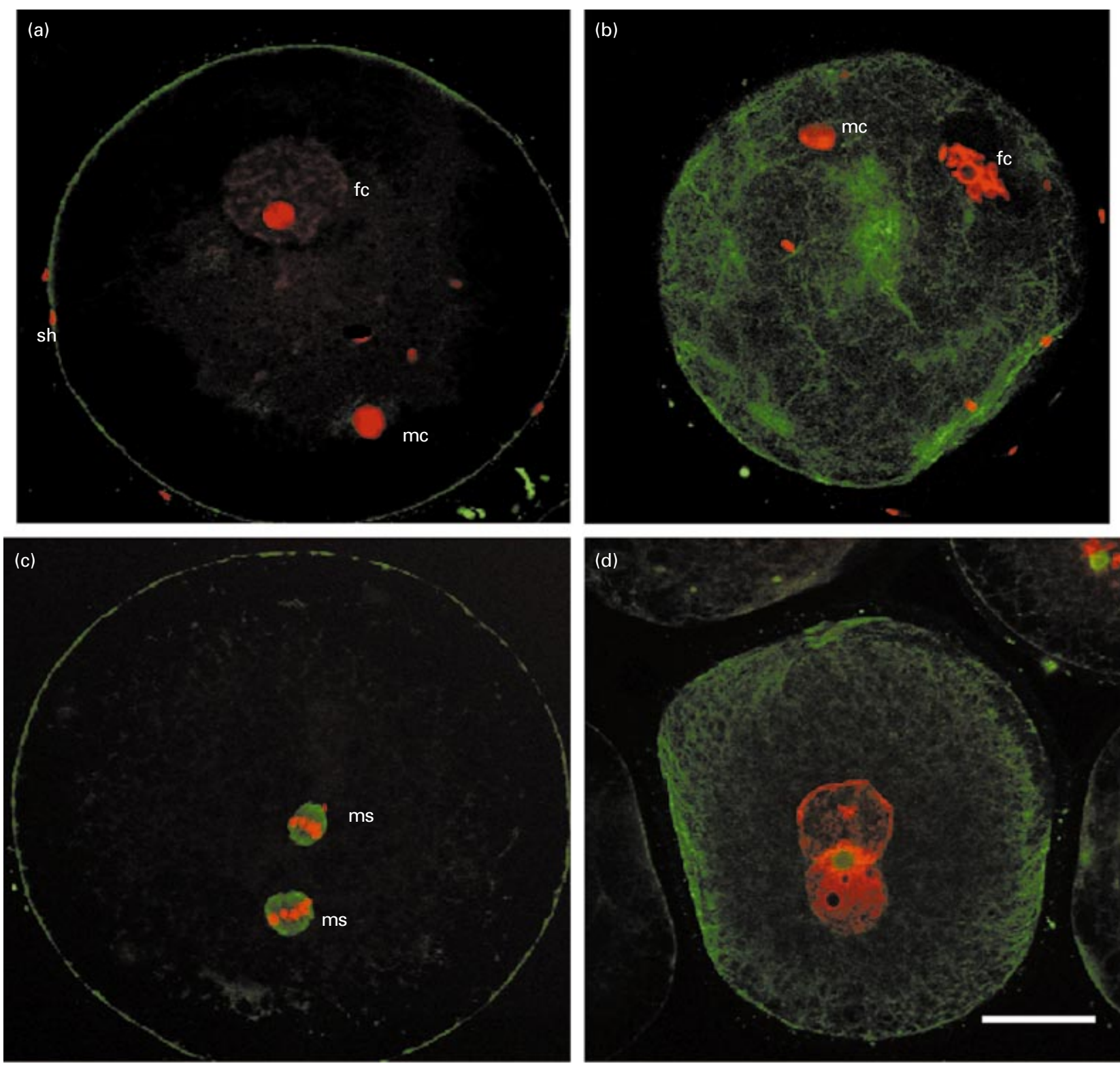

Fig. 2. Fertilized canine oocytes after in vitro culture for $72 \mathrm{~h}$. Oocytes were inseminated with fresh semen after $24 \mathrm{~h}$ of culture. (a) female chromatin $(\mathrm{fc})$ at the germinal vesicle stage and swelling male chromatin $(\mathrm{mc})$, sh: sperm head attached to the zona pellucida. (b) Female chromatin (fc) at the germinal vesicle breakdown stage and swelling male chromatin (mc) partially decondensed. (c) Fertilized oocyte at metaphase II stage. Both male and female chromatin form a metaphasic plate, each one positioned on a microtubule spindle (ms). The polar body is not visible on this scan. (d) Pronuclear stage: both male and female chromatin are moderately decondensed. Scale bar represents $30 \mu \mathrm{m}$.

a resumption of meiosis in vitro. Examination of chromatin using laser scanning confocal microscopy indicates that male chromatin undergoes marked modifications during induced meiotic maturation.

The canine oocyte has not been studied extensively due to insufficient biological material and the difficulty in determining the stage of oocyte maturation. The cytoplasm of canine oocytes is extremely rich in lipid and requires an adapted technique for the visualization of chromatin. Only a few in vivo or in vitro studies have been performed using fluorescence microscopy (Hewitt and England, 1997b; Hewitt et al., 1998). Hewitt et al. (1998) reported that the use of fluorescent DNA dyes and examination using light microscopy allowed efficient detection of germinal vesicles in immature bitch oocytes $(81-91 \%$ of visible germinal vesicles). In the present study, propidium iodide and confocal microscopy was used for the first time in bitches and proved to be a reliable technique for the visualization 


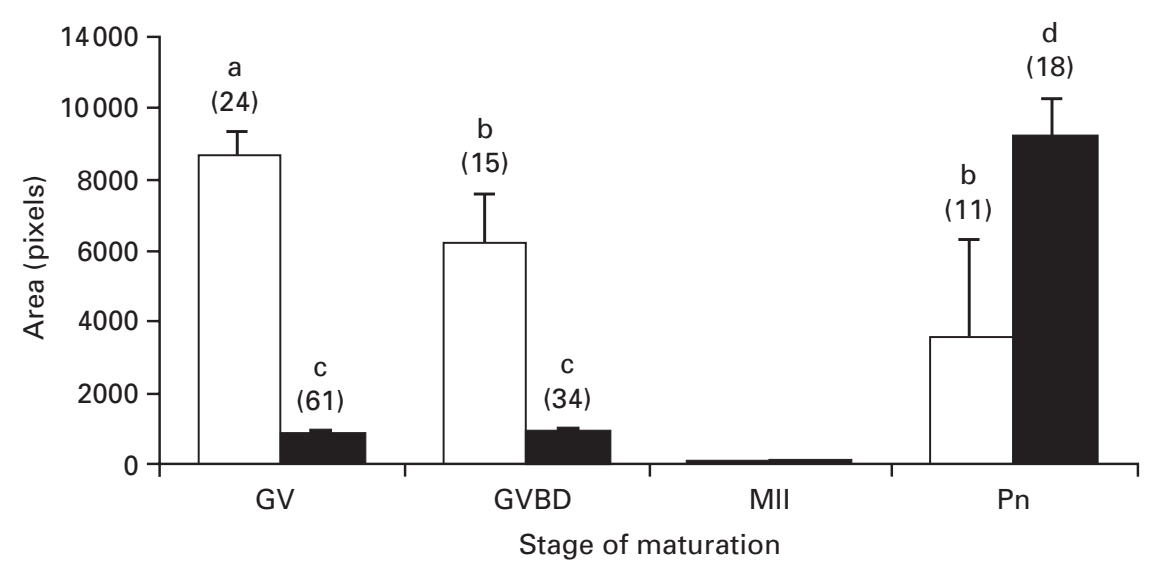

Fig. 3. Areas occupied by male ( $\square$ ) and female $(\square)$ chromatin in fertilized canine oocytes at different stages of meiotic maturation. Values are indicated as mean \pm SD (number of embryos examined). Oocytes were inseminated after $24 \mathrm{~h}$ of culture and were examined at $72 \mathrm{~h}$. For one chromatin, values with different letters are significantly different $(P<0.01)$. At metaphase II (MII), both male and female chromatin areas were considered as zero. GV: germinal vesicle; GVBD: germinal vesicle breakdown; Pn: pronuclear stage.

of chromatin in canine oocytes during meiotic maturation from the germinal vesicle stage.

In vitro maturation rates of canine oocytes are low compared with other species. In the present study, as in other studies, a high rate of degeneration $(28.9 \%)$ was observed during the entire culture period (Cinone et al., 1992: 52\%; Nickson et al., 1993: 13-55\%; Hewitt et al., 1998: 17-19\%). The observed maturation rates were very low compared with those of other domestic species: a mean of $9.6 \%$ of stages beyond metaphase I, which is comparable with the efficiency reported in anoestrous or metoestrous bitches (Cinone et al., 1992: 6\%; Robertson et al., 1992: 4-19\%; Yamada et al., 1993: 8-12\%; Hewitt et al., 1998: 12-14\%; Hewitt and England, 1999: 2-11\%). Moreover, the percentage of oocytes observed at metaphase I and II did not increase during $24-48 \mathrm{~h}$ or $48-72 \mathrm{~h}$ of culture, which confirms results reported by Robertson et al. (1992) and Yamada et al. (1993). Since bitches are receptive to mating while immature oocytes are present in the oviduct, it was hypothesized that sperm penetration plays a role in the resumption of meiosis in canine oocytes. This hypothesis was examined by performing in vitro fertilization at $24 \mathrm{~h}$ of culture and comparing maturation rates of fertilized oocytes with maturation rates of non-inseminated oocytes at $72 \mathrm{~h}$ of culture.

The data confirm that canine fertilization in vitro can occur at the germinal vesicle stage, as reported previously (Van der Stricht, 1923; Mahi and Yanagimachi, 1976; Yamada et al., 1993; Hewitt and England, 1997b). However, this observation does not confirm that fertilization occurs at the same stage in vivo, as in vitro fertilization of immature oocytes has been achieved in several species in which oocytes are ovulated at metaphase II, including small laboratory animals (Iwamatsu and Chang, 1975; Niwa and Chang, 1975; Usui and Yanagimachi, 1976; Berrios and Bedford, 1979; Cuasnicu and Bedford, 1991), cattle (Niwa et al., 1991; Chian et al., 1992), pigs (Polge and Dziuk, 1965) and humans (Van Blerkom et al., 1994). However, in most of these studies, fertilization of immature primary oocytes was abnormal: the cortical granules were not expelled (Usui and Yanagimachi, 1976; Berrios and Bedford, 1979), a much greater number of spermatozoa penetrated either the perivitelline space or the vitellus than in mature oocytes (Iwamatsu and Chang, 1972; Berrios and Bedford, 1979; Cuasnicu and Bedford, 1991) or the ooplasm was unable to decondense the sperm nucleus (Polge and Dziuk, 1965; Iwamatsu and Chang, 1972; Niwa and Chang, 1975; Usui and Yanagimachi, 1976; Berrios and Bedford, 1979; Cuasnicu and Bedford, 1991). In contrast, these abnormalities were not observed after premature fertilization in foxes, which also ovulate at the germinal vesicle stage. In foxes, the oocyte block against polyspermy, that is, the release of the cortical granule contents into the perivitelline space, may be triggered by sperm penetration at early stages of meiotic maturation (Farstad et al., 1993). Results from the present study indicate that $41.7 \%$ of fertilized oocytes at the germinal vesicle stage $(n=24)$ were polyspermic, but the average number of spermatozoa per oocyte $(3.5 \pm 1.0)$ was not significantly greater than at subsequent stages of maturation. The large number of penetrated spermatozoa may be due to the sub-optimal conditions in vitro (which are known to influence the polyspermy rate; Nagai, 1996) rather than to cytoplasmic immaturity. Furthermore, all sperm nuclei were moderately decondensed in cytoplasm of oocytes at the germinal vesicle stage. Some (unknown) sperm nuclear decondensing factors were probably already active at this 
stage. Although the data support in vitro fertilization at the germinal vesicle stage, the possibility of sperm penetration at a later stage of maturation cannot be excluded. At the time of incubation with spermatozoa, it was likely that oocytes undergoing resumption of meiosis were present.

In the present study, sperm penetration induced a resumption of meiosis in vitro. This stimulating effect of sperm penetration has already been described in molluscs and other mammals (Da-Yan and Longo, 1983; Chian et al., 1992; Van Blerkom et al., 1994). Human oocytes fertilized at the germinal vesicle stage in vitro resume meiosis until the pronuclear stage and appear to develop to the two-cell stage more quickly than oocytes fertilized at metaphase II (Van Blerkom et al., 1994). In cattle, immature oocytes can also be fertilized in vitro. In this species, only oocytes that reach metaphase I or beyond are stimulated by sperm penetration to complete meiotic maturation, whereas other oocytes remain at germinal vesicle or germinal vesicle breakdown stages after fertilization (Chian et al., 1992). Data from the present study indicate that sperm penetration has a stimulatory effect as early as the germinal vesicle stage in canine oocytes. However, the intensity of this stimulation remains to be determined. It is not possible to determine from the present study whether sperm penetration at the germinal vesicle stage induced maturation as far as metaphase II and pronuclear stages, or only until the germinal vesicle breakdown stage. Moreover, $40 \%$ of fertilized oocytes failed to resume meiosis and remained at the germinal vesicle stage. The absence of meiotic resumption in some fertilized oocytes at the germinal vesicle stage or in those that progressed only to the germinal vesicle breakdown stage does not appear to be due to an insufficient sperm stimulus as the percentage of maturation was not greater in polyspermic oocytes than in monospermic oocytes. The failure of fertilized immature oocytes to mature in vitro may reflect a lack of factors other than sperm penetration.

Fertilization at the germinal vesicle stage implies the entry of a highly compacted haploid male nucleus into the female gamete at a stage when the chromatin is partially condensed. The finding that the sperm head is moderately enlarged at the germinal vesicle stage and forms a male pronucleus when female chromatin has reached the pronuclear stage supports the observations of Yamada et al. (1992, 1993). Nevertheless, unlike Van der Stricht (1923) and Mahi and Yanagimachi (1976), parallel modification of male and female chromatin were observed during the oocyte maturation process. When the oocyte reached metaphase II, the male chromatin formed condensed chromosomes organized on a microtubule spindle. In polyspermic oocytes at metaphase II, each male nucleus formed a group of chromosomes, as reported in mouse oocytes fertilized in vitro before metaphase I (Iwamatsu and Chang, 1972; Clarke and Masui, 1987). At the pronuclear stage, both male and female chromatin were observed as largely decondensed. Thus, common cytoplasmic factors seem to transform both male and female chromatin during canine oocyte maturation. However, from the germinal vesicle stage to the germinal vesicle breakdown stage, although the female chromatin underwent condensation, a significant decrease in the area of the male chromatin was not observed.

In conclusion, sperm penetration can occur at all stages of oocyte maturation in vitro and induces a resumption of meiosis. Further studies are required to determine whether viable canine embryos can be produced in vitro using premature fertilization.

The authors extend grateful thanks to C. Petit, J. F. Salomon and $\mathrm{H}$. Quinton for their technical help. This work was supported by the French National Academy of Medicine.

\section{References}

Adenot PG, Szöllösi MS, Chesné P, Chastant S and Renard JP (1997) In vivo aging of oocytes influences the behavior of nuclei transferred to enucleated rabbit oocytes Molecular Reproduction and Development 46 325-336

Berrios M and Bedford JM (1979) Oocyte maturation: aberrant post-fusion responses of the rabbit primary oocyte to penetration by spermatozoa Journal of Cell Science 39 1-12

Chian RC, Niwa K and Nakahara H (1992) Effect of sperm penetration in vitro on completion of first meiosis by bovine oocytes arrested at various stages in culture Journal of Reproduction and Fertility 96 73-78

Cinone M, Ghein A, Caira M, Dell'Aquila ME and Minoia P (1992) Collection and maturation of oocytes in the bitch Proceedings of the $12^{\text {th }}$ International Congress on Animal Reproduction, The Hague, The Netherlands 4 1767-1769

Clarke HJ and Masui Y (1987) Dose-dependent relationship between oocyte cytoplasmic volume and transformation of sperm nuclei to metaphase chromosomes Journal of Cell Biology 104 831-840

Concannon PW, McCann JP and Temple M (1989) Biology and endocrinology of ovulation, pregnancy and parturition in the dog Journal of Reproduction and Fertility Supplement 39 3-25

Cuasnicu PS and Bedford JM (1991) Hamster oocyte penetrability during preovulatory maturation Molecular Reproduction and Development 29 $72-76$

Da-Yan C and Longo FJ (1983) Sperm nuclear dispersion coordinate with meiotic maturation in fertilized Spisula solidissima eggs Developmental Biology 99 217-224

Dedieu T, Gall L, Crozet N, Sevellec C and Ruffini S (1997) Mitogen-activated protein kinase activity during goat oocyte maturation and the acquisition of meiotic competence Molecular Reproduction and Development 45 351-358

Doak RL, Hall A and Dale HE (1967) Longevity of spermatozoa in the reproductive tract of the bitch Journal of Reproduction and Fertility $\mathbf{1 3}$ 51-58

Evans EI (1933) The transport of spermatozoa in the dog American Journal of Physiology 105 287-293

Farstad W, Hyttel P, Grondahl C, Mondain-Monval M and Smith AJ (1993) Fertilization and early embryonic development in the blue fox (Alopex lagopus) Molecular Reproduction and Development $\mathbf{3 6}$ $331-337$

Gier HT (1950) Early embryology of the dog Anatomical Record 108 $561-564$

Hewitt DA and England GCW (1997a) Effect of preovulatory endocrine events upon maturation of oocytes of domestic bitches Journal of Reproduction and Fertility $\mathbf{5 1}$ 83-91

Hewitt DA and England GCW (1997b) The canine oocyte penetration assay; its use as an indicator of dog spermatozoa performance in vitro. Animal Reproduction Science 50 123-139

Hewitt DA and England GCW (1999) Synthetic oviductal fluid and 
oviductal cell coculture for canine oocyte maturation in vitro. Animal Reproduction Science 55 63-75

Hewitt DA, Watson PF and England GCW (1998) Nuclear staining and culture requirements for in vitro maturation of domestic bitch oocytes Theriogenology 49 1083-1101

Holst PA and Phemister RD (1971) The prenatal development of the dog: preimplantation events Biology of Reproduction 5 194-206

Iwamatsu T and Chang MC (1975) Sperm penetration in vitro of mouse oocytes at various times during maturation Journal of Reproduction and Fertility 31 237-247

Kim NH, Funahashi H, Prather RS, Schatten G and Day BN (1996) Microtubule and microfilament dynamics in porcine oocytes during meiotic maturation Molecular Reproduction and Development 43 248-255

Kim NH, Chung HM, Cha KY and Chung KS (1998) Microtubule and microfilament organisation in maturing human oocytes Human Reproduction 13 2217-2222

Mahi CA and Yanagimachi R (1976) Maturation and sperm penetration of canine ovarian oocytes in vitro. Journal of Experimental Zoology 196 189-196

Nagai T (1996) In vitro maturation and fertilization of pig oocytes Animal Reproduction Science 42 153-163

Nickson DA, Boyd JS, Eckersall PD, Fergusson JM, Harvey MJA and Renton JP (1993) Molecular biological methods for monitoring oocyte maturation and in vitro fertilization in bitches Journal of Reproduction and Fertility Supplement 47 231-240

Niwa K and Chang MC (1975) Fertilisation of rat eggs in vitro at various times before and after ovulation with special references to fertilization of ovarian oocytes matured in culture Journal of Reproduction and Fertility 43 435-451

Niwa K, Park CK and Okuda K (1991) Penetration in vitro of bovine oocytes during maturation by frozen-thawed spermatozoa Journal of Reproduction and Fertility 91 329-336

Pearson OP and Enders RK (1943) Ovulation, maturation and fertilization in the fox Anatomical Record 85 69-83

Polge C and Dziuk P (1965) Recovery of immature eggs penetrated by spermatozoa following induced ovulation in the pig Journal of Reproduction and Fertility 9 357-358

Renton JP, Boyd JS, Eckersall PD, Fergusson JM, Harvey MJA, Mullaney J and Perry B (1991) Ovulation, fertilization and early embryonic development in the bitch (Canis familiaris) Journal of Reproduction and Fertility 93 221-231

Robertson JB, Srsen V and King WA (1992) Cytogenetic and ultrastructural analysis of canine oocytes cultured in vitro. Proceedings of the $12^{\text {th }}$ International Congress on Animal Reproduction, The Hague, The Netherlands 4 1808-1810

Sutovsky P and Schatten G (1998) Ultrastructural aspects of mammalian fertilization: new discoveries and inspirations from the work of Daniel Szöllösi Reproduction, Nutrition and Development 38 629-641

Tsutsui T (1989) Gamete physiology and timing of ovulation and fertilization in dogs Journal of Reproduction and Fertility Supplement 39 269-275

Tsutsui T and Shimazu Y (1975) Studies on the reproduction in the dog Japanese Journal of Animal Reproduction 21 65-75

Usui N and Yanagimachi R (1976) Behaviour of hamster sperm nuclei incorporated into eggs at various stages of maturation, fertilization and early development Journal of Ultrastructure Research 57 276-288

Van Blerkom J, Davis PW and Merriam J (1994) The developmental ability of human oocytes penetrated at the germinal vesicle stage after insemination in vitro. Human Reproduction 9 697-708

Van der Stricht $\mathbf{O}$ (1923) Etude comparée des ovules des mammifères aux différentes périodes de l'ovogenèse Archives de Biologie 33 229-300

Yamada S, Shimazu Y, Kawaji H, Nakazawa M, Naito K and Toyoda Y (1992) Maturation, fertilization and development of dog oocytes in vitro. Biology of Reproduction 46 853-858

Yamada S, Shimazu Y, Kawano Y, Nakazawa M, Naito K and Toyoda Y (1993) In vitro maturation and fertilization of preovulatory dog oocytes Journal of Reproduction and Fertility Supplement 47 227-229

Received 2 May 2000

Accepted 7 August 2000 